\title{
Low-dose metformin improves pregnancy rate in in vitro fertilization repeaters without polycystic ovary syndrome: Prediction of effectiveness by multiple parameters related to insulin resistance
}

\author{
Masao Jinno $^{1,2}$, Kenichi Kondou ${ }^{1}, K_{0 j i}$ Teruya $^{3}$ \\ ${ }^{1}$ Department of Obstetrics and Gynecology, School of Medicine, Kyorin University, Mitaka City, ${ }^{2}$ Women's Clinic \\ Jinno, Choufu City, ${ }^{3}$ Department of Public Health, School of Health Sciences, Kyorin University, Hachioji City, Tokyo, \\ Japan
}

\begin{abstract}
OBJECTIVE: Insulin resistance is associated with aging and stress, both common among patients repeatedly failing to conceive with in vitro fertilization (IVF repeaters). In the present study we examined whether low-dose metformin could improve the outcome in IVF repeaters without polycystic ovary syndrome (PCOS). DESIGN: Study I was a preliminary clinical trial aiming at defining indications for therapy; study II was a prospective randomized study. The studies involved a university hospital and a private infertility clinic. We studied 232 women without PCOS who had failed at least twice to conceive by previous IVF. Metformin (500 mg/ day) was administered for 8 to 12 weeks before and during ovarian stimulation (metformin IVF). In study I, IVF outcomes with metformin $(n=33)$ were compared to outcomes without metformin of previous IVF in the same subjects. A discriminant score (DS) was determined from nine parameters assessed before metformin administration to predict achievement of ongoing pregnancy by metformin IVF. In study II $(n=199)$, ongoing pregnancy rates were compared prospectively between groups with/without metformin and with DS above/below 0.6647 . RESULTS: Study I. Ongoing pregnancy rate improved significantly with metformin compared with previous IVF, and pregnancy correlated significantly with a DS at an optimal threshold of 0.6647 (sensitivity, 0.90; specificity, 0.91). Study II. Ongoing pregnancy and implantation rates were significantly higher in women with a DS above 0.6647 who received metformin $(56 \%$ and 33\%) compared with those having a DS below 0.6647 with metformin (14\% and $11 \%)$ and those having a DS above/below 0.6647 without metformin $(20 \%$ and $7.1 \% / 15 \%$ and $11 \%$, respectively). CONCLUSIONS: Low-dose metformin improved pregnancy rate in IVF repeaters without PCOS, probably by decreasing insulin resistance. Indication can be determined from insulin-resistance-related multiple parameters assessed before metformin administration.
\end{abstract}

Key words: Assisted reproductive technology, Insulin resistance, Insulin sensitizers, IVF repeaters, Metformin, Pregnancy 


\section{INTRODUCTION}

Various methods for ovarian stimulation have been developed to enhance oocyte number and quality as well as endometrial receptivity. As a result, a satisfactory rate of pregnancy in most IVF patients has been achieved. ${ }^{1}$ However, a number of patients resistant to conventional methods are still encountered in clinical practice, including poor responders (who develop few or no oocytes even with full ovarian stimulation), ${ }^{2}$ older women, especially those in their $40 \mathrm{~s},{ }^{3}$ and women who for unknown reasons develop sufficiently numerous but poor-quality oocytes. ${ }^{4}$ Therefore, further development of methods for ovarian stimulation is required.

Insulin resistance is pivotal in the pathogenesis of polycystic ovary syndrome (PCOS), ${ }^{5}$ which has been treated successfully by metformin, an insulin-sensitizing agent, aiming to improve rates of ovulation and pregnancy. ${ }^{6,7}$ Insulin resistance is also encountered in various conditions such as aging, ${ }^{8}$ stress $^{9}$ depression, ${ }^{10}$ obesity, ${ }^{11}$ and sedentary life-style. ${ }^{12}$ These conditions frequently exist in infertile women, suggesting a pathogenic role of insulin resistance even in nonPCOS patients. Consistently, non-PCOS women with ovarian dysfunction more often have dysfunction in the stress-response-mechanism in terms of abnormal circadian rhythm of cortisol and elevated catecholamine levels, compared with normal volunteers. ${ }^{13}$ It has in fact been demonstrated that the prevalence of insulin resistance is significantly higher among non-PCOS infertile women with oligomenorrhea than among those with eumenorrhea. ${ }^{14}$ We therefore hypothesized that in non-PCOS patients who repeatedly fail to conceive by IVF, the administration of metformin could improve ovarian function and IVF outcome by restoring insulin sensitivity.

First, we performed a prospective clinical trial (study I) to examine the effect of low-dose metformin on IVF outcome in patients without PCOS, who had repeatedly failed to conceive in previous IVF attempts (IVF repeaters). In this group we analyzed the results to define indications for metformin therapy. Next, we performed a prospective randomized study (study II) to confirm the effectiveness of low-dose metformin therapy in IVF repeaters without PCOS.

\section{SUBJECTS AND METHODS}

\section{Study I}

In this prospective clinical trial, we included 33 infertile women without PCOS who had failed to conceive in two or more previous IVF attempts. PCOS was excluded according to the 2003 Rotterdam criteria. ${ }^{15}$ The causes of infertility were ovarian dysfunction, tubal factors, endometriosis, male factors, ovarian dysfunction + male factors, tubal + male factors, or unexplained cause in 11, 4, 1, 1, 9, 2, and 5 women, respectively. Eleven women always showed poor ovarian response, i.e., development of less than three follicles, with full ovarian stimulation in their previous IVF attempts. Thirteen women had an age of 40 years or more.

On day 3 of a spontaneous menstrual cycle, physical examination [body mass index (BMI), blood pressure (BP)], various blood drawings, hormone measurements, and a 75-g oral glucose tolrance test (OGTT) were carried out between 09:00 and 12:00 after 12 $\mathrm{h}$ of fasting. (Laboratory methods are described below under "Clinical examinations"). Then daily administration of metformin (Melbin; Sumitomo Pharmaceutical, Tokyo, Japan) was begun at the dose of $250 \mathrm{mg}$ daily, and increased at weekly intervals by $250 \mathrm{mg}$, to a final dose of 500 to $750 \mathrm{mg}$ per day. After 4 to 6 weeks of metformin administration, physical examination and laboratory testing were carried out on day 3 of the cycle. At 8 to 12 weeks of metformin therapy, patients underwent the long protocol of gonadotropin-releasing hormone $(\mathrm{GnRH})$ agonist and human menopausal gonadotropin (hMG) administration for ovarian stimulation in preparation for IVF or intracytoplasmic sperm injection (ICSI).

Clinical pregnancy was diagnosed by ultrasonographic observation of a gestational sac, and an ongoing pregnancy was a gestation extending beyond 16 weeks, including those progressing to delivery. Following either delivery or diagnosis of non-pregnacy, administration of metformin was terminated after one week at a tapered dose. The data from metformin patients with either IVF or ICSI were combined (metformin IVF/ICSI group), since no significant differences in the rates of fertilization, embryonic development, or pregnancy were noted between the 
two fertilization procedures.

We then compared follicular development, fetilization, embryonic development, and pregnancy rates in the metformin IVF/ICSI group with those in previous IVF/ICSI attempts without metformin in the same subjects.

The results of various examinations before administration of metformin were compared between patients whose treatment with metformin and IVF/ ICSI resulted in ongoing pregnancy and those whose treatment concluded with failure (i.e. non-pregnancy or spontaneous abortion). The results were also compared with results after metformin administration. Using parameters selected by these comparisons, we attempted to predict achievement of ongoing pregnancy in metformin plus IVF/ICSI treatment by discriminant analysis.

Based on the data of study I we determined that a discriminant score (DS) calculated from nine parameters present before administration of metformin highly correlated with achievement of ongoing pregnancy in the metformin IVF/ICSI group; the rate of ongoing pregnancy was significantly higher in women with a DS of at least 0.6647 than in those with a lower DS.

\section{Study II}

Study II included a new consecutive series of 199 IVF repeaters without PCOS. In this prospective study the rates of ongoing pregnancy were compared among four groups defined by DS (above $v s$. below 0.6647) and metformin treatment vs. without. All subjects had failed to conceive in two or more previous IVF attempts without metformin, and none had been enrolled in study I. As in study I, subjects underwent multiple examinations on day 3 of a spontaneous cycle; they were then prospectively assigned at random to undergo ovarian stimulation either with or without low-dose metformin. Randomization used sealed envelopes and did not consider DS scores. Protocols for metformin administration and ovarian stimulation were the same as in study I.

Eighteen and 80 women receiving metformin had a DS above and below 0.6647 (groups A and B), respectively, while 15 and 86 women not receiving metformin had a DS above and below 0.6647 (groups
C and D), respectively.

Informed consent was obtained from all subjects. The study was approved by the Kyorin University Ethics Committee.

\section{Ovarian stimulation, in vitro fertilization, and intracytoplasmic sperm injection}

Follicular development was stimulated with the "long protocol," involving GnRH agonist and hMG administration, with or without administration of metformin as described above. Nasal administration of buserelin acetate (Suprecur; Hoechst, Tokyo, Japan), $900 \mu \mathrm{g}$ per day, was begun on day 4 of the luteal phase preceding the IVF/ICSI cycle, and continued at the same dose until administration of human chorionic gonadotropin (hCG). Daily administration of three ampules of hMG (Pergonal; Teikokuzouki, Tokyo, Japan; 75 IU of LH plus 75 IU of FSH per ampule) was begun between days 3 and 10 of the follicular phase in the IVF/ICSI cycle. Serum concentrations of $17 \beta$-estradiol were measured every morning. When dominant follicles reached $14 \mathrm{~mm}$ in diameter, the dose of hMG was reduced to 2 ampules per day. Human chorionic gonadotropin (Gonatropin, Teikokuzouki, Tokyo, Japan), 10,000 IU, was administered when one or more follicles were at least $18 \mathrm{~mm}$ in diameter and the serum $17 \beta$-estradiol concentration exceeded 300 $\mathrm{pg} / \mathrm{mL}$ (conversion factor to SI units, 3.671).

Oocytes were collected transvaginally $36 \mathrm{~h}$ after hCG administration. Semen was diluted two times with human tubal fluid (HTF) medium (no. 9962; Irvine Scientific, Irvine, CA) containing $10 \%$ patient serum. ${ }^{16}$ Diluted semen was centrifuged directly if semen analysis results were normal, or after placement upon two layers of Sil-Select solutions (FertiPro NV, Beemem, Belgium) if analysis results were abnormal. Semen analyses were performed according to World Health Organization criteria. ${ }^{17}$ The sperm pellet was resuspended in medium and then centrifuged again, after which motile spermatozoa were collected by a swim-up technique. Oocytes were inseminated 2 to $6 \mathrm{~h}$ after their retrieval at a concentration of 80,000 motile spermatozoa per mL. ICSI was performed when the partner had severe male infertility with a sperm count less than $5 \times 10^{6}$ per $\mathrm{mL}$ and/or motility in fewer than $20 \%$ of sperm.

Oocytes were considered to be fertilized when 
two pronuclei were observed at 17 to $19 \mathrm{~h}$ following insemination or ICSI. Embryos were transferred to the uterus 38 to $48 \mathrm{~h}$ following insemination. Morphologically high-quality embryos were defined as grades 1 and 2 according to Veeck's criteria. ${ }^{18}$ A 25 -mg dose of progesterone was administered daily throughout the luteal phase.

\section{Clinical examinations before and after metformin administration}

Between 09:00 and 12:00 (after at least $12 \mathrm{~h}$ of fasting), all patients underwent various examinations on day 3 of a spontaneous menstrual cycle prior to and 4 to 6 weeks after metformin administration. These included BMI, systolic (S) and diastolic (D) BP, glucose metabolism assessment including 75-g OGTT, homeostasis model assessment-insulin resistance index [HOMA-R = fasting plasma glucose $(\mathrm{FPG}) \mathrm{x}$ immunoreactive insulin during fasting (FIRI)/405], hemoglobin $(\mathrm{Hb}) \mathrm{A} 1 \mathrm{c}$, lipid metabolism assessment [total cholesterol (TC), high-density lipoprotein cholesterol (HDL), low-density lipoprotein cholesterol (LDL), triglyceride (TG)], purine metabolism assessment, [uric acid (UA)], hepatic enzymes (ALP, $\gamma$-GTP, LDH, LAP, GOT, and GPT), renal function assessment: [blood urea nitrogen (BUN), creatinine, electrolytes ( $\mathrm{Na}, \mathrm{K}, \mathrm{Cl}$, and $\mathrm{Ca})$ ]. Total blood count, and endocrinologic assessments: LH, FSH, PRL, testosterone, free testosterone, dehydroepiandrosterone sulfate (DHEA-S), and 17 $\beta$-estradiol, and urinalysis were also determined.

Serum insulin and plasma glucose were measured by enzyme immunoassay (EIA, Lumipulse Insulin; Fuji Rebio, Tokyo, Japan) and amperometry using a GOD-fixation enzyme membrane and a peroxidizing electrode, respectively. ALP and $\gamma$-GTP were measured using a Quick Autoneo ALP-JS II Kit and a Quick Autoneo $\gamma$-GT Kit (Sinotest, Tokyo, Japan), respectively. Sensitivities and, in parentheses, intraand interassay coefficients of variation were 0.05 $\mu \mathrm{U} / \mathrm{mL}(2.1 \%, 1.8 \%)$ for insulin; $0.1 \mathrm{mg} / \mathrm{dL}(1.0 \%$, $1.0 \%)$ for glucose; $7 \mathrm{IU} / \mathrm{L}(0.51 \%, 0.29 \%)$ for ALP; and $0.1 \mathrm{IU} / \mathrm{L}(0.80 \%, 0.65 \%)$ for $\gamma-\mathrm{GTP}$, respectively.

Serum concentrations of $17 \beta$-estradiol and testosterone were measured with radioimmunoassay kits from Diagnostic Products, Los Angeles, CA. Sensitivities and, in parentheses, intra- and interassay coefficients of variation were $10 \mathrm{pg} / \mathrm{mL}(5.6 \%, 6.8 \%)$ for $17 \beta$-estradiol and $5 \mathrm{ng} / \mathrm{dL}(5.2 \%, 2.3 \%)$ for testosterone, respectively.

\section{Statistical analysis}

Data were not distributed normally, except the discriminant score. Data were analyzed using MannWhitney U-test, Wilcoxon test, Kruscal-Wallis test, the chi-squared test, Fisher's exact test, receiver-operating characteristics (ROC) curve analysis, multiple logistic regression analysis, or discriminant analysis as appropriate. The discriminant score were compared by analysis of variance (ANOVA) and then Fisher's protected least significant difference (PLSD) test. $\mathrm{p}$ values less than 0.05 were considered to indicate significance. Results are presented as the mean \pm SEM unless otherwise stated.

\section{RESULTS}

\section{Study I}

Age, BMI, and numbers of previous failed IVF/ ICSI attempts in the subjects were $37.5 \pm 0.8$ years, $23.1 \pm 0.5 \mathrm{~kg} / \mathrm{m}^{2}$, and $3.2 \pm 0.4$, respectively.

Follicular development and IVF/ICSI outcomes were compared between metformin IVF/ICSI patients and their own previous IVF/ICSI attempts without metformin (Table 1). Follicular development was enhanced significantly in the metformin IVF/ICSI group in terms of dominant follicle diameter, serum $17 \beta$-estradiol levels, and $17 \beta$-estradiol divided by the number of follicles of at least $12 \mathrm{~mm}$ in diameter on the day of hCG administration. Endometrial thickness was similar in the two groups while the number of retrieved oocytes, fertilized oocytes, and embryos showing cleavage appeared to be higher in the metformin IVF/ICSI compared with previous IVF/ICSI, although the differences did not achieve statistical significance. The number of morphologically high-grade embryos was significantly higher in the metformin IVF/ICSI than in the previous IVF/ICSI.

The most dramatic improvement with metformin therapy was observed in the rates of clinical and ongoing pregnancies, which were significantly higher with metformin IVF/ICSI than in the patients' previous IVF/ICSI (Table 1). 
Table 1. IVF/ICSI outcomes in women without PCOS and with repeated IVF/ICSI failures, on metformin compared to outcomes of previous attempts without metformin in the same subjects

\begin{tabular}{|c|c|c|}
\hline & $\begin{array}{c}\text { Metformin } \\
\text { IVF/ICSI }\end{array}$ & $\begin{array}{l}\text { Previous IVF/ } \\
\text { ICSI }\end{array}$ \\
\hline Stimulated cycles & 33 & 104 \\
\hline $\begin{array}{l}\text { Dominant follicle diameter } \\
(\mathrm{mm})^{\mathrm{a}}\end{array}$ & $19.6 \pm 0.2^{b}$ & $18.6 \pm 0.2$ \\
\hline $\begin{array}{l}\text { No. of follicles } \geq 12 \mathrm{~mm} \text { in } \\
\text { diameter }^{\mathrm{a}}\end{array}$ & $12.6 \pm 1.8$ & $10.8 \pm 0.9$ \\
\hline $\operatorname{Estradiol}(\mathrm{pg} / \mathrm{mL})^{\mathrm{a}}$ & $2418 \pm 276^{c}$ & $1878 \pm 144$ \\
\hline $\begin{array}{l}\text { Estradiolper follicle } \geq 12 \mathrm{~mm} \\
\quad(\mathrm{pg} / \mathrm{mL})^{\mathrm{a}}\end{array}$ & $252 \pm 18^{b}$ & $208 \pm 10$ \\
\hline Endometrial thickness $(\mathrm{mm})^{\mathrm{a}}$ & $10.9 \pm 0.3$ & $10.3 \pm 0.2$ \\
\hline $\begin{array}{l}\text { Canceled cycles ( } \% \text { of } \\
\text { stimulated cycles) }\end{array}$ & $0(0 \%)$ & $9(8.7 \%)$ \\
\hline Oocyte retrieval (cycles) & 33 & 95 \\
\hline Embryo transfer & 33 & 94 \\
\hline $\begin{array}{l}\text { Clinical pregnancy (\% of } \\
\text { stimulated cycles) }\end{array}$ & $12(36.4 \%)^{\mathrm{d}}$ & $10(9.6 \%)$ \\
\hline $\begin{array}{l}\text { Ongoing pregnancy (\% of } \\
\text { stimulated cycles) }\end{array}$ & $10(30.3 \%)^{\mathrm{e}}$ & $2(1.9 \%)$ \\
\hline No. of oocytes & $11.5 \pm 1.6$ & $9.3 \pm 1.0$ \\
\hline No. of fertilized oocytes & $8.1 \pm 1.1$ & $6.5 \pm 0.7$ \\
\hline No. of embryos & $7.6 \pm 1.0$ & $6.2 \pm 0.6$ \\
\hline No. of superior embryos & $3.2 \pm 0.5^{\mathrm{c}}$ & $2.1 \pm 0.2$ \\
\hline
\end{tabular}

${ }^{\mathrm{a}}$ On the day of hCG administration.

${ }^{b}$ Mean \pm SEM, $\mathrm{p}<0.01$ vs. previous IVF/ICSI (Mann-Whitney u-test).

${ }^{c} \mathrm{p}<0.05$ vs. previous IVF/ICSI (Mann-Whitney u-test).

${ }^{\mathrm{d}} \mathrm{p}<0.001$ vs. previous IVF/ICSI (chi-squared test).

${ }^{\mathrm{e}} \mathrm{p}<0.0001$ vs. previous IVF/ICSI (Fisher's exact test).

Various parameters before administration of metformin were compared between patients whose metformin IVF/ICSI resulted in ongoing pregnancies $(n=10)$ and patients who failed (i.e. non-pregnancy or spontaneous abortion, $n=23$ ). Patients with successful outcome were significantly younger than failing patients $(34.4 \pm 1.7 v s .38 .9 \pm 0.8$ years; $\mathrm{p}<0.05$, Mann-Whitney U-test). Patients with failure despite metformin had lower pretreatment values than those achieving ongoing pregnancy in FIRI $(7.8 \pm 0.8 v s$. 10.6 $\pm 1.3 \mu \mathrm{U} / \mathrm{mL}, \mathrm{p}<0.05$, Mann-Whitney U-test), HOMA-R $\left(1.7 \pm 0.2\right.$ vs. $\left.2.4 \pm 0.3 \mathrm{mcUg} / \mathrm{L}^{2}, \mathrm{p}<0.05\right)$, and ALP (109.4 $\pm 6.6 v s .133 .3 \pm 9.5 \mathrm{IU} / \mathrm{L}, \mathrm{p}<0.05)$. In a multiple logistic regression analysis of five factors (age, FPG, FIRI, HOMA-R, and number of failed previous IVF/ICSI attempts), significant predictors of pregnancy achievement with metformin IVF/ICSI were age $(\mathrm{p}<0.05$, Odds ratio: $0.45,95 \%$ confidence interval: 0.24 to 0.84 ), FPG ( $p<0.052 .7,1.1$ to 6.7 ) and number of previous failed IVF/ICSI attempts $(\mathrm{p}<0.05,3.5,1.1$ to 10.5$)$.

Physical and laboratory parameters were also compared between time points before and after 4 to 6 weeks of metformin administration. Metformin administration significantly decreased SBP (from $127.7 \pm 3.2$ to $121.8 \pm 2.3 \mathrm{~mm} \mathrm{Hg}, \mathrm{p}<0.05$, Wilcoxon test), DBP (from $79.5 \pm 2.0$ to $75.8 \pm 1.5 \mathrm{~mm} \mathrm{Hg}, \mathrm{p}<0.05$ ), testosterone (from $0.26 \pm 0.02$ to $0.20 \pm 0.02 \mathrm{ng} / \mathrm{dL}, \mathrm{p}<0.05$ ), LAP (from $24.6 \pm 0.6$ to $23.5 \pm 0.6 \mathrm{IU} / \mathrm{L}, \mathrm{p}<0.05$ ), $\mathrm{LDH}$ (from $345.6 \pm 12.0$ to $317.3 \pm 7.8 \mathrm{IU} / \mathrm{L}, \mathrm{p}<0.05$ ), total serum protein concentration (from $7.5 \pm 0.1$ to $7.3 \pm 0.1 \mathrm{~g} / \mathrm{dL}, \mathrm{p}<0.05$ ), and hematocrit (from $38.7 \pm 0.6$ to $38.1 \pm 0.6 \%, \mathrm{p}<0.05)$.

We then attempted to predict achievement of ongoing pregnancy with metformin IVF/ICSI by discriminant analysis using 9 parameters obtained before administration of metformin (age, BMI, number of previous failed IVF/ICSI attempts, FPG, FIRI,

Table 2. Canonical discriminant function coefficients to predict achievement of ongoing pregnancy by metformin IVF/ICSI

\begin{tabular}{lc}
\hline $\begin{array}{l}\text { Parameters before metformin } \\
\text { administration (Unit) }\end{array}$ & Coefficients \\
\hline Age (years) & -0.349 \\
Body mass index $\left(\mathrm{kg} / \mathrm{m}^{2}\right)$ & 0.064 \\
Number of previous failed IVF/ICSI & 0.455 \\
$\quad$ attempts & \\
Fasting plasma glucose (mg/dL) & 0.248 \\
Fasting immunoreactive insulin (FIRI, & 1.314 \\
$\mu \mathrm{U} / \mathrm{mL})$ & \\
HOMA-R & -6.111 \\
Systolic blood pressure (mmHg) & 0.037 \\
ALP $(\mathrm{IU} / \mathrm{L})$ & 0.005 \\
$\gamma$-GTP $(\mathrm{IU} / \mathrm{L})$ & 0.052 \\
(Constant) $^{\mathrm{a}}$ & -17.536
\end{tabular}

${ }^{a}$ Homeostasis model assessment-insulin resistance index.

${ }^{\mathrm{b}}$ Alkaline phosphatase.

${ }^{c} \gamma$-Glutamyl transpeptidase.

Glucose: To convert to SI units multiply by 0.0555

Insulin: To convert to SI units multiply by 7.175 
HOMA-R, SBP, ALP, and $\gamma$-GTP). Canonical discriminant function coefficients of these parameters were determined and are shown in Table 2. The discriminant score (DS) is defined as (the sum of each parameter multiplied by its coefficient) - 17.536, and if the DS is greater than or equal to a cut-off value, achievement of ongoing pregnancy can be predicted. The optimal cut-off value was determined by a ROC curve (Figure 1) and was 0.6647 . The result of discriminant analysis using this optimal cut-off value is as follows: of 11 patients with a DS greater than or equal to $0.6647,9$ had ongoing pregnancies with metformin IVF/ICSI (positive predictive value, 0.82); of 22 patients with a DS less than $0.6647,21$ had failure (negative predictive value, 0.95; sensitivity, 0.90 and specificity, 0.91; $\mathrm{p}<0.001$, Fisher's exact test).

\section{Study II}

Between 98 and 101 women receiving ovarian stimulation with and without metformin respectively, no significant difference (Mann-Whitney U-test and

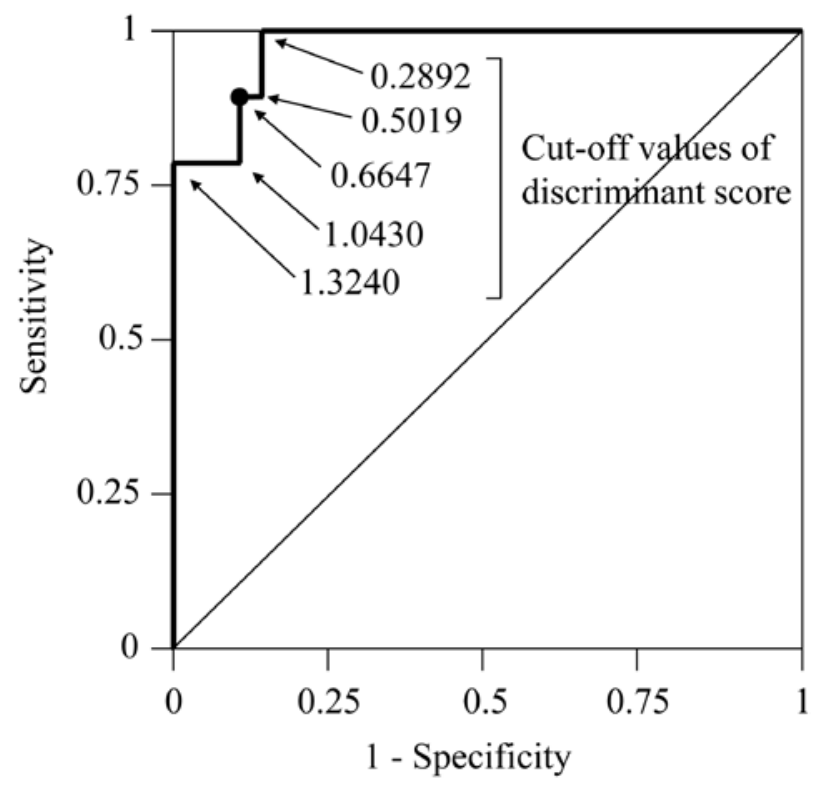

Figure 1. Receiver-operating-characteristics (ROC) analysis to determine a discriminant score (DS) for prediction of ongoing pregnancy with metformin IVF/ICSI. The DS is defined as the sum of each parameter multiplied by its coefficient (Table 2) -17.536; if the DS is greater than or equal to a cut-off value, achievement of ongoing pregnancy can be predicted. A cutoff value of 0.6647 for the DS, which corresponds to the point nearest to the coordinates on the ROC curve (1-specificity $=0$, sensitivity $=1$ ), was chosen as the optimal cut-off value. the chi-squared test) was observed with respect to age (39.2 \pm 0.4 vs $39.1 \pm 0.4$ years), parity number $(0.14 \pm 0.04$ vs $0.14 \pm 0.04)$, BMI $\left(21.5 \pm 0.2\right.$ vs $\left.21.3 \pm 0.2 \mathrm{~kg} / \mathrm{m}^{2}\right)$, day- 3 FSH (11.4 \pm 0.7 vs $10.5 \pm 0.6 \mathrm{IU} / \mathrm{L})$, or distribution of causes of infertility (ovarian dysfunction, tubal factors, endometriosis, male factors, ovarian dysfunction + male factors, tubal + male factors, or unexplained: $37,10,3,1,26,10$, and 11 women, respectively for the metformin group and 43, 13, 2, 3, 24, 8, and 8 women, respectively for the no-metformin group).

To confirm effectiveness of low-dose metformin therapy and the DS as an indication in IVF repeaters without PCOS, IVF/ICSI outcomes were compared prospectively between groups $\mathrm{A}, \mathrm{B}, \mathrm{C}$, and $\mathrm{D}$, as defined by DS above or below 0.6647 and treatment with or without metformin (Table 3). No significant differences (Kruscal-Wallis test) were seen between the four groups in numbers of superior embryos (Table $3)$ and transferred embryos per retrieval $(3.1 \pm 0.3$, $2.9 \pm 0.2,3.7 \pm 0.3$, and $3.0 \pm 0.2$ in groups $\mathrm{A}, \mathrm{B}, \mathrm{C}$, and $\mathrm{D}$, respectively). However, rates of clinical and ongoing pregnancy were significantly higher in group A, compared with any of the other groups (Table 3). Rates of implantation and fetal viability per transferred embryo also were significantly higher in group A than the other groups.

Thus, low-dose metformin improved the rate of ongoing pregnancy in $20 \%$ of IVF repeaters without PCOS; these metformin-responsive women could be anticipated if DS is above 0.6647 .

\section{DISCUSSION}

This study demonstrated that administration of low-dose metformin can improve rates of implantation and ongoing pregnancy in about $20 \%$ of women without PCOS who have repeatedly failed to conceive in previous IVF attempts (IVF repeaters). Metformin appeared to be effective in women with relatively high FPG and FIRI, and increased BP and serum concentrations of testosterone and hepatic enzymes. Metformin-responsive IVF repeaters without PCOS could be identified by a DS value above 0.6647 , calculated from nine parameters assessed before administration of metformin: age, BMI, number of previously failed IVF/ICSI attempts, FPG, FIRI, HOMA-R, SBP, ALP, and $\gamma$-GTP. 
Table 3. IVF/ICSI outcomes among the four groups of non-PCOS IVF/ICSI repeaters: with and without merformin and according to the DS value (above or below 0.6647 )

\begin{tabular}{lcccc}
\hline & \multicolumn{2}{c}{ With metformin } & \multicolumn{2}{c}{ Without metformin } \\
\cline { 2 - 5 } Discriminant score (Group) & $\begin{array}{c}\mathbf{0 . 6 6 4 7} \\
(\mathbf{A})\end{array}$ & $\begin{array}{c}<\mathbf{0 . 6 6 4 7} \\
\mathbf{( B )}\end{array}$ & $\begin{array}{c}\mathbf{0 . 6 6 4 7} \\
\text { (C) }\end{array}$ & $\begin{array}{c}<\mathbf{0 . 6 6 4 7} \\
\text { (D) }\end{array}$ \\
\hline No. of women & 18 & 80 & 15 & 86 \\
Discriminant score & $1.83 \pm 0.27^{\mathrm{a}}$ & $-1.67 \pm 0.15$ & $1.71 \pm 0.31^{\mathrm{a}}$ & $-1.89 \pm 0.16$ \\
Oocyte retrievals & 18 & 80 & 15 & 86 \\
No. of superior embryos per retrieval & $3.4 \pm 0.8$ & $2.5 \pm 0.4$ & $3.8 \pm 0.8$ & $2.7 \pm 0.3$ \\
Clinical pregnancies (\% of oocyte retrieval) & $10(56 \%)^{\mathrm{b}}$ & $16(20 \%)$ & $4(27 \%)$ & $22(26 \%)$ \\
Ongoing pregnancies (\% of oocyte retrieval) & $10(56 \%)^{\mathrm{c}}$ & $11(14 \%)$ & $3(20 \%)$ & $13(15 \%)$ \\
Transferred embryos & 55 & 226 & 56 & 260 \\
Gestational sacs (\% of transferred embryo) & $18(33 \%)^{\mathrm{d}}$ & $25(11 \%)$ & $4(7.1 \%)$ & $28(11 \%)$ \\
Viable fetus (\% of transferred embryo) & $13(24 \%)^{\mathrm{e}}$ & $16(7.1 \%)$ & $4(7.1 \%)$ & $16(6.2 \%)$ \\
\hline
\end{tabular}

${ }^{a}$ Mean \pm SEM, $p<0.0001$ vs. groups B and D (ANOVA and Fisher's PLSD).

${ }^{b} \mathrm{p}<0.01$ vs. group B (Fisher's exact test) and $\mathrm{p}<0.05$ vs. group $\mathrm{D}$ (chi-squared test).

${ }^{c} p<0.001$ vs. groups B and D (Fisher's exact test) and $p<0.05$ vs. group $C$ (chi-squared test).

${ }^{\mathrm{d}} \mathrm{p}<0.0001$ vs. groups $\mathrm{B}$ and $\mathrm{D}$ (qui-square test) and $\mathrm{p}<0.001$ vs. group $\mathrm{C}$ (chi-squared test).

${ }^{\mathrm{e}} \mathrm{p}<0.0001, \mathrm{p}<0.001$ and $\mathrm{p}<0.05$ vs. groups D, B and C, respectively (chi-squared test).

Insulin resistance and compensatory hyperinsulinemia are known to play major pathogenetic roles in various diseases, particularly type 2 diabetes mellitus..$^{19}$ Evidence has accumulated that selective insulin resistance and subsequent hyperinsulinemia are pivotal in the pathogenesis of PCOS as well. ${ }^{5,20}$ In PCOS, administration of metformin reduces insulin resistance, serum concentration of insulin and androgen, and $\mathrm{BP},{ }^{21,22}$ and improves rates of ovulation and pregnancy. ${ }^{6,21,22}$ Hypertension is associated with insulin resistance and hyperinsulinemia, ${ }^{23}$ while administration of metformin increases insulin sensitivity and decreases BP. ${ }^{24}$ In nonalcoholic fatty liver disease, also considered a manifestation of insulin resistance syndrome, ${ }^{25,26}$ elevations of serum GOT, GPT, ALP, and $\gamma$-GTP are commonly detected..$^{25,26}$ Obesity, defined as an excessive BMI or waist-to-hip ratio, is well known to cause insulin resistance. ${ }^{11}$

Results of the present discriminant analysis suggested that IVF repeaters with higher pretreatment values for BMI, FPG, FIRI, SBP, ALP, or $\gamma$-GTP are more likely to benefit from metformin therapy. Elevation of any of these parameters is associated with insulin resistance. Thus, the mechanism by which metformin improved outcome in IVF repeaters in the present study appeared to involve improved insulin sensitivity, as is true for metformin therapy in PCOS. ${ }^{5,20,21}$ Consistently, patients achieving ongoing pregnancy with metformin had significantly higher pretreatment values of FIRI and HOMA-R than those with failure, suggesting that metformin is more effective in women with lower insulin sensitivity. Moreover, our observations that metformin significantly decreased BP, serum androgen, and serum hepatic enzymes in IVF repeaters provided further support for this mechanism. The tendency for increasing efficacy of metformin with increasing number of previous failed IVF/ICSI attempts may be explained as follows: repeated IVF/ICSI failures by conventional ovarian stimulation without metformin resulted in selection of women with insulin resistance requiring metformin treatment.

Although the subjects of the present study did not have PCOS according to the 2003 Rotterdam criteria, it cannot be excluded that some women might have less overt PCOS or a predisposition for PCOS given the high prevalence, great variability of symptoms, and lack of consensus concerning the definition of PCOS. ${ }^{27}$ Most likely, however, decreased insulin sensitivity, which is caused by multiple factors such 
as aging, stress, anxiety, depression, obesity, and sedentary lifestyle, common in IVF repeaters may be present in these patients. A progressive decline in insulin sensitivity with advancing age is widely known. ${ }^{8}$ Chronic stress which causes dysregulation of stress response systems, mainly the hypothalamic-pituitaryadrenocortical (HPA) axis and the sympathoadrenal system (SAS), can promote insulin resistance. ${ }^{9,11}$ Moreover, stressful daily events, trait anxiety, and depressed mood have been linked with increased cortisol secretion, ${ }^{28,29}$ followed by insulin resistance and central obesity. ${ }^{11}$ Obesity and sedentary habits ${ }^{12}$ are well-known contributors to insulin resistance.

In the present study, the beneficial effect of metformin on pregnancy rate was more evident than on follicular and embryonic development, suggesting that metformin may improve not only oocyte quality but also endometrial receptivity. Support to this notion is offered by the combined results of metformin IVF/ICSI in studies I and II in which the rate of abortion was significantly lower in women with DS above 0.6647 ( $0 \%$ in 19 clinical pregnancies) than in those below 0.6647 (26\% in 19 clinical pregnancies, $\mathrm{p}<0.05$, Fisher's exact test). This speculation may also be supported by an observation that metformin administration during pregnancy reduced first-trimester pregnancy loss in women with PCOS..$^{30,31}$ Moreover, it has been reported that metformin administration increases serum concentrations of glycodelin, a putative marker of endometrial function, and those of insulin-like growth factor-binding protein-1 in the luteal phase; it also enhanced uterine vascularity and blood flow. ${ }^{32}$

The beneficial effect of metformin on IVF outcome using gonadotropin stimulation has been shown in women with PCOS, ${ }^{33-38}$ but this was not detected in other ${ }^{39,40}$ studies. The study by Fedorcsak ${ }^{34}$ included PCOS patients with insulin resistance, while three studies $^{33,37,38}$ examined PCOS patients with high fasting insulin $(24.2 \pm 1.9 \mu \mathrm{U} / \mathrm{mL}, 16 \pm 3.8$ and $10.1 \pm 6.4$, respectively), values suggestive of insulin resistance. On the other hand, the two negative reports ${ }^{39,40}$ included PCOS patients without consideration of insulin resistance. Metformin is probably more effective in an insulin-resistant population of IVF patients with PCOS.
To assure optimal effects of metformin, an appropriate dose of metformin should be chosen according to the degree of insulin resistance. A major mechanism by which metformin produces beneficial metabolic effects is activation of AMP-activated protein kinase, which the drug induces in a concentration-dependent manner. ${ }^{41}$ In PCOS patients treated with metformin at a widely used dose range, 1000 to $1500 \mathrm{mg}$ per day, the total number of developing follicles was decreased with no change in follicles larger than 14 $\mathrm{mm}$ in diameter, resulting in an increased number of mature oocytes. ${ }^{33,35}$ However, in non-PCOS IVF repeaters treated with the same dose of metformin in a preliminary investigation, follicular development was further suppressed, decreasing the number of mature oocytes (from $4.1 \pm 0.8$ without metformin to $2.3 \pm 0.7$ with $1500 \mathrm{mg}$ metformin per day, $\mathrm{n}=5$, unpublished data). In the present study, the exclusion of women with PCOS and the inclusion of IVF repeaters might have eliminated many patients with overt and no insulin resistance. Consequently, our study was likely to include a significant number of subjects with mild insulin resistance readily corrected by low-dose metformin (500 mg per day), resulting in improvement of IVF outcomes. Our DS conceivably identified such women with mild insulin resistance, resulting in higher rates of pregnancy with low-dose metformin IVF in this subgroup.

This is the first report to show that low-dose metformin enhanced IVF pregnancy rates in women without PCOS. The population responsive to this therapy was identified by a combination of parameters associated with the insulin resistance syndrome. Considering insulin resistance as a factor not only for glycemic control but also for various organ functions affected by insulin action may further clarify the role of insulin sensitivity in fertility problems not related to PCOS.

\section{REFERENCES}

1. de Mouzon J, Lancaster P, 1997 World collaborative report on in vitro fertilization, preliminary data for 1995. J Assisted Reprod Genet, 14: Suppl: 250-265.

2. Tarlatzis BC, Zepiridis L, Grimbizis G, Bontis J, 2003 Clinical management of low ovarian response to stimulation for IVF: a systematic review. Hum Reprod Update, 9: 61-76. 
3. Tarlatzis BC, Zepiridis L, 2003 Perimenopausal conception. Ann NY Acad Sci 997: 93-104.

4. Jinno M, Katsumata Y, Hoshiai T, Nakamura Y, Matsumoto K, Yoshimura Y, 1997 A therapeutic role of prolactin supplementation in ovarian stimulation for in vitro fertilization: the bromocriptine-rebound method. J Clin Endocrinol Metab 82: 3603-3611.

5. Dunaif A, 1997 Insulin resistance and the polycystic ovary syndrome: mechanism and implications for pathogenesis. Endocr Rev 18: 774-800.

6. Costello MF, Eden JA, 2003 A systematic review of the reproductive system effects of metformin in patients with polycystic ovary syndrome. Fertil Steril 79: 1-13.

7. Fleming R, 2006 The use of insulin sensitizing agents in ovulation induction in women with Polycystic Ovary Syndrome. Hormones (Athens) 5: 171-178.

8. Paolisso G, Tagliamonte MR, Rizzo MR, Giugliano D, 1999 Advancing age and insulin resistance: new facts about an ancient history. Eur J Clin Invest 29: 758-769.

9. Vanltallie TB, 2002 Stress: a risk factor for serious illness. Metabolism 51: 40-45.

10. Wolkowitz OM, Epel ES, Reus VI, 2001 Stress hormone-related psychopathology: pathophysiological and treatment implications. World J Biol Psychiatry 2: 115-143.

11. Bjorntorp P, Rosmond R, 2000 The metabolic syndrome - a neuroendocrine disorder? Br J Nutr 83:S49-S57.

12. Rosenthal M, Haskell WL, Solomon R, Widstrom A, Reaven GM, 1983 Demonstration of a relationship between levels of physical training and insulin-stimulated glucose utilisation in normal humans. Diabetes 32: 408-411.

13. Jinno M, Watanabe A, Takahashi S, Urakami C, 2007 A novel method to detect dysfunction of stress-response-mechanism in women with ovarian dysfunction: implications of circadian rhythm in salivary cortisol for diagnosis and treatment. Fertil Steril 88: Suppl 1: S171.

14. Ohgi S, Nakagawa K, Kojima R, Ito M, Horikawa T, Saito H, 2008 Insulin resistance in oligomenorrheic infertile women with non-polycystic ovary syndrome. Fertil Steril 90: 373-377.

15. The Rotterdam ESHRE/ASRM-sponsored PCOS consensus workshop group, 2004 Revised 2003 consensus on diagnostic criteria and longterm health risks related to polycystic ovary syndrome (PCOS). Hum Reprod 19: 41-47.

16. Jinno M, 1986 Comparison of media used for human in vitro fertilization and embryo transfer programs: a new method of serum preparation. Acta Obst Gynaec Jpn 38: 102-110.

17. World Health Organization, 1987 WHO Laboratory Manual for Examination of Human Semen and SemenCervical Mucus Interaction. Cambridge: Cambridge University Press; pages 1-64.
18. Scott RT, Hofmann GE, Veeck LL, Jones HW Jr, Muasher SJ, 1991 Embryo quality and pregnancy rates in patients attempting pregnancy through in vitro fertilization. Fertil Steril 55: 426-428.

19. Reaven GM, 1988 Role of insulin resistance in human disease. Diabetes 37: 1595-1607.

20. Zacur HA, 2001 Polycystic ovary syndrome, hyperandrogenism, and insulin resistance. Obstet Gynecol Clin North Am 28: 21-33.

21. Velazquez EM, Mendoza S, Hamer T, Sosa F, Glueck CJ, 1994 Metformin therapy in polycystic ovary syndrome reduces hyperinsulinemia, insulin resistance, hyperandrogenemia, and systolic blood pressure, while facilitating normal menses and pregnancy. Metabolism 43: 647-654.

22. Nestler JE, Jakubowicz DJ, Evans WS, Pasquali R, 1998 Effects of metformin on spontaneous and clomipheneinduced ovulation in the polycystic ovary syndrome. $\mathrm{N}$ Engl J Med 338: 1876-1880.

23. Ferrannini E, Buzzigoli G, Bonadonna R, et al 1987 Insulin resistance in essential hypertension. $\mathrm{N}$ Engl $\mathrm{J}$ Med 317: 350-357.

24. Landin K, Tengborn L, Smith U, 1991 Treating insulin resistance in hypertension with metformin reduces both blood pressure and metabolic risk factors. J Intern Med 229: 181-187.

25. Patrick L, 2002 Nonalcoholic fatty liver disease: relationship to insulin sensitivity and oxidative stress. Treatment approaches using vitamin E, magnesium, and betaine. Altern Med Rev 7: 276-291.

26. Zafrani ES, 2004 Non-alcoholic fatty liver disease: an emerging pathological spectrum. Virchows Arch 444: 3-12.

27. Balen A, Michelmore K, 2002 What is polycystic ovary syndrome? Are national views important? Hum Reprod 17: 2219-2227.

28. Eck MV, Berkhof H, Nicolson N, Sulon J, 1996 The effects of perceived stress, traits, mood states, and stressful daily events on salivary cortisol. Psychosom Med 58: 447-458.

29. Smyth J, Ockenfels MC, Porter L, Kirschbaum C, Hellhammer DH, Stone AA, 1998 Stressors and mood measured on a momentary basis are associated with salivary cortisol secretion. Psychoneuroendocrinology 23: 353-370.

30. Jakubowicz DJ, Iuorno MJ, Jakubowicz S, Roberts KA, Nestler JE, 2002 Effects of metformin on early pregnancy loss in the polycystic ovary syndrome. $\mathrm{J}$ Clin Endocrinol Metab 87: 524-529.

31. Essah PA, Cheang KI, Nestler JE, 2004 The Pathophysiology of miscarriage in women with polycystic ovary syndrome. Review and proposed hypothesis of mechanism involved. Hormones (Athens) 3: 221-224.

32. Jakubowicz DJ, Seppala M, Jakubowicz S, et al 2001 Insulin reduction with metformin increases luteal phase serum glycodelin and insulin-like growth factor-binding 
protein 1 concentrations and enhances uterine vascularity and blood flow in the polycystic ovary syndrome. $\mathrm{J}$ Clin Endocrinol Metab 86: 1126-1133.

33. Stadtmauer LA, Toma SK, Riehl RM, Talbert LM, 2001 Metformin treatment of patients with polycystic ovary syndrome undergoing in vitro fertilization improves outcomes and is associated with modulation of the insulin-like growth factors. Fertil Steril 75: 505-509.

34. Fedorcsak P, Dale PO, Storeng R, Abyholm T, Tanbo T, 2003 The effect of metformin on ovarian stimulation and in vitro fertilization in insulin-resistant women with polycystic ovary syndrome: an open-label randomized cross-over trial. Gynecol Endocrinol 17: 207-214.

35. Doldi N, Persico P, Sebastiano FD, Marsiglio E, Ferrari A, 2006 Gonadotropin-releasing hormone antagonist and metformin for treatment of polycystic ovary syndrome patients undergoing in vitro fertilization-embryo transfer. Gynecol Endocrinol 22: 235-238.

36. Tang T, Glanville J, Orsi N, Barth JH, Balen AH, 2006 The use of metformin for women with PCOS undergoing IVF treatment. Hum Reprod 21: 1416-1425.
37. Qublan HS, Al-Khaderei S, Abu-Salem AN, et al 2009 Metformin in the treatment of clomiphene citrate-resistant women with polycystic ovary syndrome undergoing in vitro fertilisation treatment: A randomised controlled trial. J Obstet Gynaecol 29: 651-655.

38. Kumbak B, Kahraman S, 2009 Efficacy of metformin supplementation during ovarian stimulation of lean PCOS patients undergoing in vitro fertilization. Acta Obstet Gynecol Scand 88: 563-568.

39. Kjotrod SB, von During V, Carlsen SM, 2004 Metformin treatment before IVF/ICSI in women with polycystic ovary syndrome; a prospective, randomized, double blind study. Hum Reprod 19: 1315-1322.

40. Onalan G, Pabuccu R, Goktolga U, Ceyhan T, Bagis T, Cincik M, 2005 Metformin treatment in patients with polycystic ovary syndrome undergoing in vitro ferilization: a prospective randomized trial. Fertil Steril 84: 798-801.

41. Zhou G, Myers R, Li Y, et al 2001 Role of AMP-activated protein kinase in mechanism of metformin action. J Clin Invest 108: 1167-1174. 